\title{
Epileptic seizure-related changes in electrocorticogram, cortical temperature, and cerebral hemodynamics obtained via an implantable multimodal multichannel probe during preoperative monitoring: illustrative case
}

\author{
Manami Wakuya, BS, ${ }^{1}$ Takao Inoue, $\mathrm{PhD},{ }^{2}$ Hirochika Imoto, MD, $\mathrm{PhD},{ }^{3}$ Yuich Maruta, $\mathrm{PhD},{ }^{3}$ Sadahiro Nomura, MD, $\mathrm{PhD},{ }^{3}$ \\ Michiyasu Suzuki, $\mathrm{MD}, \mathrm{PhD}{ }^{2}$ and Toshitaka Yamakawa, $\mathrm{PhD}^{1}$ \\ ${ }^{1}$ Department of Computer Science and Electrical Engineering, Graduate School of Science and Technology, Kumamoto University, Kumamoto, Kumamoto, Japan; and \\ Departments of ${ }^{2}$ Advanced ThermoNeuroBiology and ${ }^{3}$ Neurosurgery, Yamaguchi University School of Medicine, Ube, Yamaguchi, Japan
}

\begin{abstract}
BACKGROUND Electrocorticography (EcoG) plays an essential role in the preoperative evaluation of epilepsy, despite its high invasiveness. Brain temperature and cerebral hemodynamics also reflect brain activity. This study examined whether a multimodal multichannel probe that simultaneously records EcoG, cortical temperature, and cerebral hemodynamics can contribute to improving the assessment of epileptic seizures. After preoperative monitoring was performed in a patient with epilepsy, three generalized seizures and two focal seizures were observed.

OBSERVATIONS A short-term power increase in the alternating current spectrogram, high-amplitude slow waves in direct current potential, an increase in cortical temperature, an increase in oxyhemoglobin $\left(\mathrm{HbO}_{2}\right)$ concentration and total hemoglobin $(\mathrm{HbT})$ concentration, and a decrease in deoxyhemoglobin $(\mathrm{HHb})$ concentration, followed by a decrease in $\mathrm{HbO}_{2}$ and $\mathrm{HbT}$ concentrations and an increase in $\mathrm{HHb}$ concentration, were observed in generalized seizures. However, no changes in these pathophysiological signals were observed in focal seizures.
\end{abstract}

LESSONS Seizure-related changes regarding generalized seizures were consistent with the results of previous studies. The results of generalized and focal seizures indicate that epileptic brain activity propagated from the epileptic focus in the right frontal lobe to the measurement area near the motor cortex in generalized seizures but not in focal seizures.

https://thejns.org/doi/abs/10.3171/CASE21694

KEYWORDS electrocorticography; near-infrared spectroscopy; cortical temperature; multimodal probe; subdural measurement

In the preoperative evaluation of epilepsy, electrocorticography $(E c o G)$ directly recorded from the cortical surface or brain parenchyma is a basic procedure, ${ }^{1,2}$ and it still plays an essential role ${ }^{3}$ despite its highly invasive nature.

However, it has become clear that epileptic brain activity can be observed through various pathophysiological signals. The direct current (DC) potential indicates brain activity below $0.5 \mathrm{~Hz}$, which shows gradual changes. Slow baseline shifts in DC potential associated with epileptic seizures were observed by subdural and scalp recordings in a human study. ${ }^{4}$ In addition, brain temperature elevation due to epileptic seizures was observed by the simultaneous measurement of EcoG and brain temperature in rats ${ }^{5}$ and mice. ${ }^{6}$ Near-infrared spectroscopy (NIRS) is a method used to obtain changes in the concentration of oxyhemoglobin $\left(\mathrm{HbO}_{2}\right)$, deoxyhemoglobin $(\mathrm{HHb})$, and total hemoglobin $(\mathrm{HbT})$ using the transmission property of near-infrared rays in living tissues. Concurrent EcoG and NIRS measurements elucidated changes in cerebral hemodynamics related to epileptic activity. Changes in cerebral hemodynamics around the onset and end of epileptic seizures have been observed in rats $^{7}$ and children. ${ }^{8}$ Furthermore, NIRS helped identify seizures with subtle ictal electroencephalography (EEG) abnormalities, which were missed during scalp EEG inspection in simultaneous scalp EEG

ABBREVIATIONS AC = alternating current; CBF = cerebral blood flow; ch = channel; $D C=$ direct current; EcoG = electrocorticography;

$\mathrm{EEG}=$ electroencephalography; $\mathrm{FS}=$ focal seizure; $\mathrm{GS}=$ generalized seizure; $\mathrm{HbO}_{2}=$ oxyhemoglobin; $\mathrm{HbT}=$ total hemoglobin; $\mathrm{HHb}=$ deoxyhemoglobin;

LED = light-emitting diode; NIRS = near-infrared spectroscopy; PD = photodiode.

INCLUDE WHEN CITING Published March 7, 2022; DOI: 10.3171/CASE21694.

SUBMITTED December 10, 2021. ACCEPTED January 20, 2022.

(C) 2022 The authors, CC BY-NC-ND 4.0 (http://creativecommons.org/licenses/by-nc-nd/4.0/). 
and NIRS recordings. ${ }^{9}$ Thus, multimodal probes that simultaneously monitor EcoG, cortical temperature, and cerebral hemodynamics were considered to be effective for improving the assessment of epileptic seizures.

However, there are several issues with the conventional studies. Brain temperature and cerebral hemodynamics are known to fluctuate in relation to epileptic seizures, but few have been measured directly from the human brain surface. As for the change in brain temperature, both temperature elevation and reduction were described in previous studies. Brain temperature elevation due to epileptic seizures has been observed in rats ${ }^{5}$ and mice. ${ }^{6}$ However, another study suggested the possibility of a decrease in brain temperature in humans, in contrast to rats. ${ }^{10}$ In addition, hemodynamic changes induced by epileptic seizures vary among studies. Some studies observed an increase in $\mathrm{HbO}_{2}$ and $\mathrm{HbT}$ with a decrease in $\mathrm{HHb}$ in rats ${ }^{11}$ and in patients with epilepsy. ${ }^{12}$ Other studies reported that cerebral hemodynamics alternated between an increase in $\mathrm{HbO}_{2}$ accompanied by a decrease in $\mathrm{HHb}$ and a decrease in $\mathrm{HbO}_{2}$ accompanied by an increase in $\mathrm{HHb}$ around the onset of a seizure and returned to the baseline in epileptic rats ${ }^{7}$ and patients with epilepsy. ${ }^{8,13}$

In a previous study, the pathological neural activity in patients with epilepsy during surgery and ruptured aneurysmal subarachnoid hemorrhage of the postoperative state was monitored using the proposed probe. ${ }^{14}$ In this study, preoperative monitoring was performed in a patient with epilepsy who also had cortical dysplasia using the proposed probe, and the changes in the EcoG, cortical temperature, and cerebral hemodynamics during focal seizures (FSs) and generalized seizures (GSs) were clarified.

\section{Illustrative Case}

\section{Fabrication of Multimodal Probe}

The proposed probe was designed to measure EcoG, cortical temperature, and cerebral hemodynamics simultaneously, with an outer shape similar to that of the strip electrode, commonly used in neurosurgery, as shown in Fig. $1 \mathrm{~A}$. The dimensions of the probe head are $10 \mathrm{~cm}$ (length), $8 \mathrm{~mm}$ (width), and $0.7 \mathrm{~mm}$ (maximum total thickness). Flexible printed circuit technology was used so that the probe head had a suitable thickness for subdural implantation, and the probe could be mass produced for disposable use.

The proposed probe measures each modality in six channels. For the EcoG measurement, six 3-mm-diameter platinum electrodes were arranged at $1-\mathrm{cm}$ intervals. For cortical temperature, negative temperature coefficient thermistors (ERTJZEG103FA, $10 \mathrm{k} \Omega$ at $25^{\circ} \mathrm{C}, 0.6 \times 0.3 \times 0.3 \mathrm{~mm}$; Panasonic) were mounted next to the EcoG electrodes. For cerebral hemodynamics, two types of infrared light-emitting diode (LED) bare chips that emit different wavelengths (C770-40P for $770 \mathrm{~nm}$ and C810-40P for $810 \mathrm{~nm}, 0.4 \times 0.4 \times$ $0.25 \mathrm{~mm}$; Epitex) and a photodiode (PD) bare chip that works as a receiver of the emitted near-infrared rays (PD2501, $1.3 \times 3.1 \times$ $0.3 \mathrm{~mm}$; Epitex) were used. The change in hemoglobin concentration was determined through NIRS. In addition, to measure the changes in the hemoglobin concentration in the same cortical region as the EcoG and cortical temperature, these LEDs and the PD were located on either side of the platinum electrode along with a thermistor for each channel (Fig. 1B).

After the assembly of the circuit was completed, all electronic components were sealed with nontoxic transparent silicone. Then, all the components and substrates, except for the EcoG electrode, were coated with a $10-\mu m$-thick Parylene-C layer. These procedures improved the insulation and biocompatibility during chronic implantation and removed the acute toxicity from the surface of the proposed probe.

\section{Preoperative Monitoring of Epilepsy Resection Surgery}

The patient was a 31-month-old female with epilepsy accompanied by cortical dysplasia in the right frontal lobe. The measurement was performed before the epilepsy resection surgery. Subdural electrodes used for diagnosis were implanted in the right frontal lobe to cover the epileptic focus, whereas the multimodal probe was installed near the right motor cortex to avoid any interference with the diagnosis, as shown in Fig. $1 \mathrm{C}$ and D. The subdural electrodes were monitored using a video EEG monitoring system (EEG-1200A, Nihon Kohden Co., Ltd.) with a sampling frequency of $2 \mathrm{kHz}$. For the data acquired from the multimodal probe, two EEG amplifiers (EBM-1016, Unique Medical Co., Ltd.) were connected to the EcoG electrodes in parallel to record the alternating current (AC) and DC potentials. For the DC potential, the EcoG signals were passed through a $1-\mathrm{kHz}$ low-pass filter. The filtered EcoG, thermistor signal, NIRS signal, and another pathophysiological signal (electrocardiogram) were simultaneously collected at a sampling frequency of $200 \mathrm{~Hz}$ by two digitizers (AIO-163202FX-USB, Contec Co., Ltd.). These pathophysiological signals were stored on a computer using data acquisition software (LabDAQ5-CT Multi, Matsuyama Advance Co., Ltd.). For the AC potential, the EcoG signals were collected at a sampling frequency of $2 \mathrm{kHz}$ using a physiological data acquisition device (PowerLab 8/35, AD Instruments) and were recorded using software (LabChart $8, A D$ Instruments). Monitoring was conducted for approximately 55 hours, during which $A C$, $\mathrm{DC}$, thermistor, and NIRS signals were recorded simultaneously.

\section{Postmonitoring Processing}

Seizure onset was determined by a clinical neurophysiology technologist on the basis of the video EEG data. The DC potential was extracted from the EcoG signal using a $0.01-\mathrm{Hz}$ low-pass filter. The thermistor and NIRS signals were calculated using a method described in a previous study. ${ }^{14}$ A Butterworth high-pass filter was adapted to the DC, thermistor, and NIRS signals to remove artifacts. Their cutoff frequencies were $0.0025,0.001$, and $0.0025 \mathrm{~Hz}$, respectively. Conversely, each value from the $D C$, thermistor, and NIRS signals was subtracted by each baseline to show signals without the Butterworth filter on a scale similar to the filtered signals. The baseline of the DC and NIRS signals was considered as a median from 10 to 5 minutes before the onset. However, a median ranging from 2.5 minutes before the onset to 2.5 minutes after the onset was used as a baseline in the thermistor signal because a change in cortical temperature related to an epileptic seizure is extremely minimal compared with the baseline fluctuation. After these procedures, the calculated thermistor signals were smoothed using the moving average of a 10-second time window to further refine the quantization steps of the waveform caused by the resolution of the thermistor signal converter. Calculations and signal processing were performed using MATLAB (version 9.5).

\section{Results}

Three GSs (GS1-3) and two FSs (FS1 and FS2) were observed during the monitoring. The changes in AC spectrogram, DC, cortical temperature, and cerebral hemodynamics for GS1, GS2, and GS3, which were obtained through the multimodal probe and processed using the Butterworth filter, are shown in Figs. 2-4. The changes in 

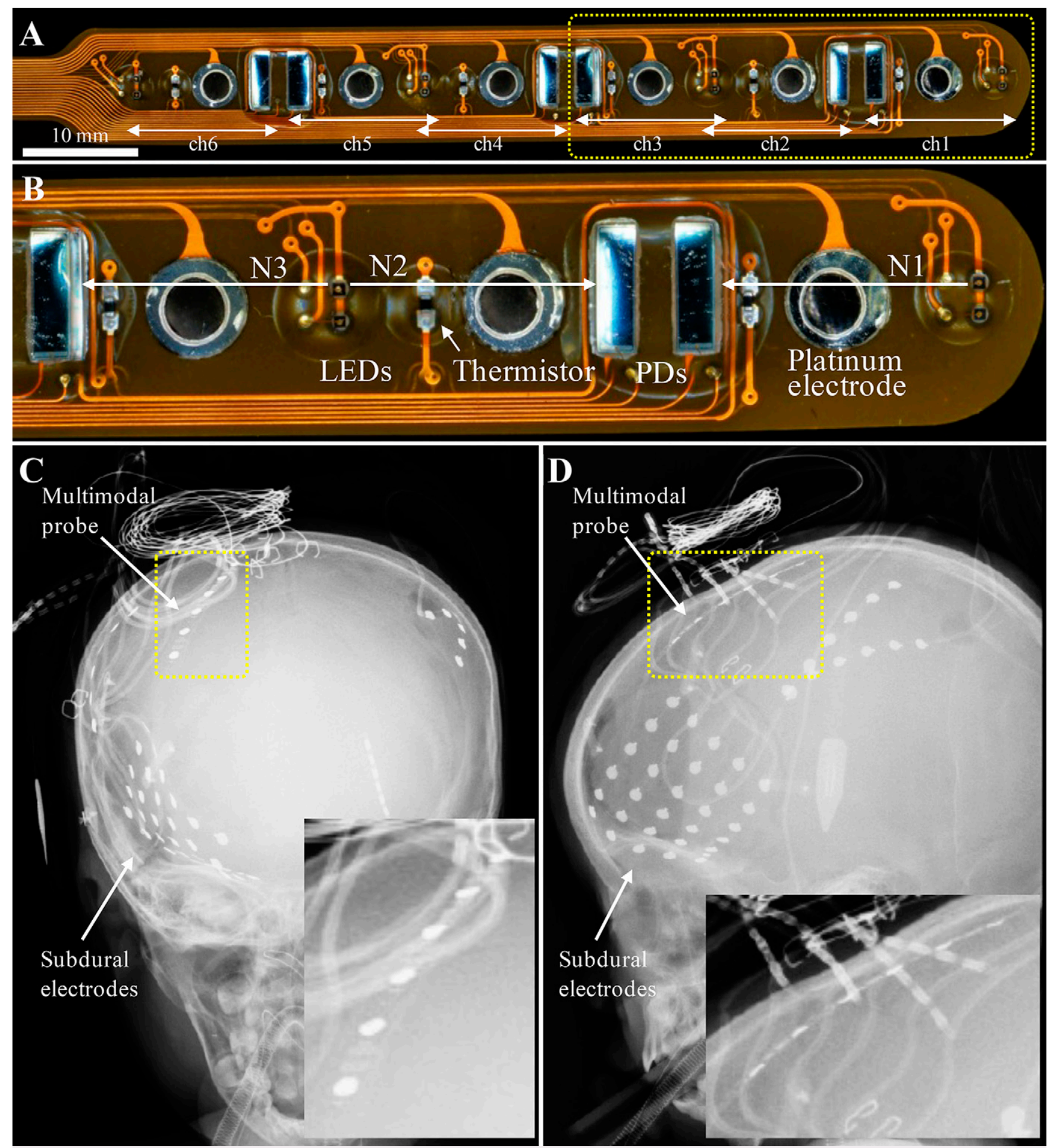

FIG. 1. Microphotographs of the multimodal probe and radiographic images after the electrode implantation surgery. A: Probe head has six channels, which measure EcoG, cortical temperature, and cerebral hemodynamics. White arrows show the distribution of ch1-6. B: Enlarged view of the yellow dotted area. Each channel is composed of a platinum electrode, LEDs, a PD, and a thermistor. White arrows indicate the path of infrared rays from the LEDs to a PD for cerebral hemodynamics and the number corresponding to that channel. Frontal view (C) and lateral view (D) of radiographic images. Subdural electrodes used for diagnosis were installed around the epileptic focus in the right frontal lobe, whereas a multimodal probe was placed near the right motor cortex to avoid interference with the diagnosis. The area around the proposed probe is indicated by the yellow dotted square and magnified in the lower right corner of each figure. 

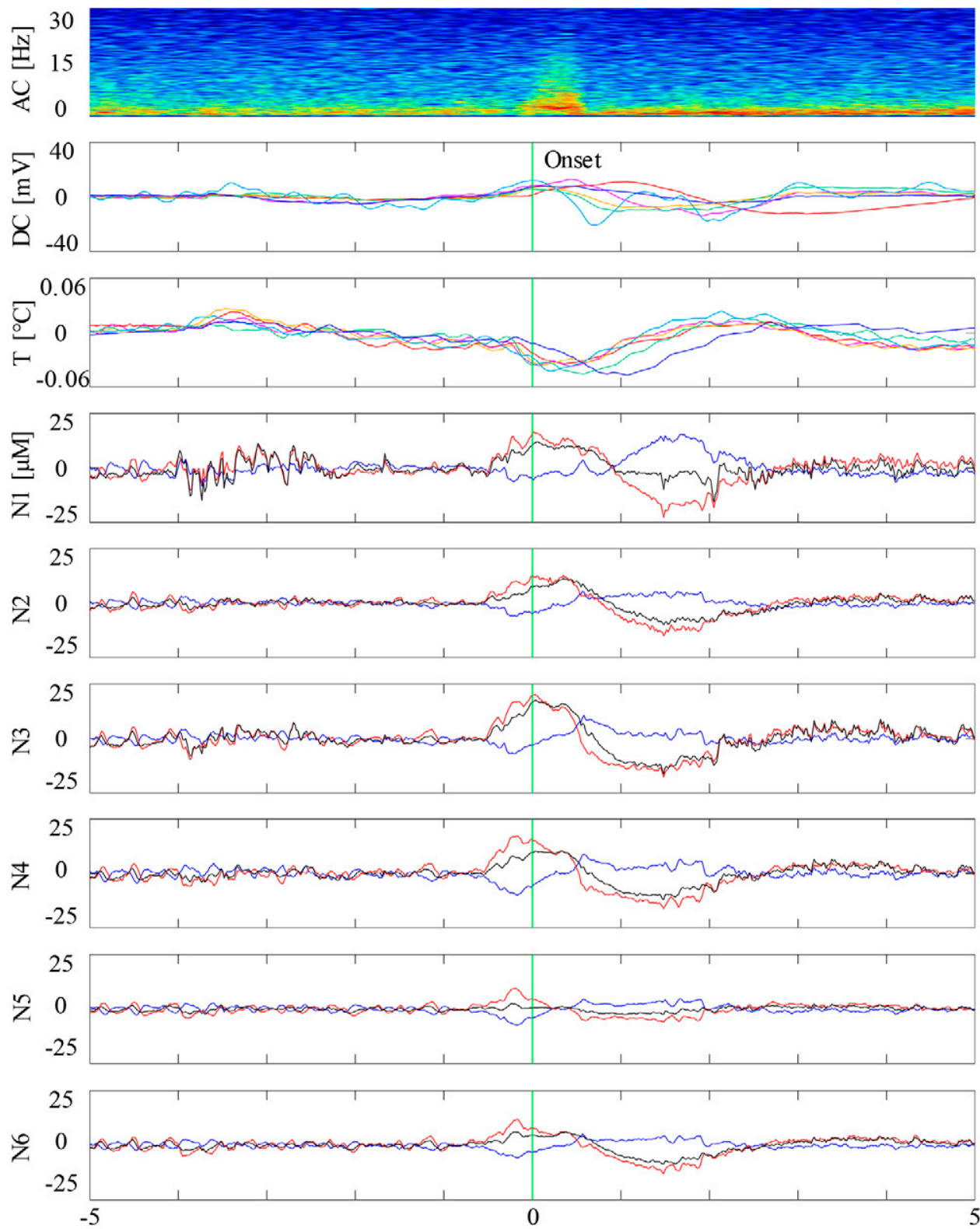

Time $[\mathrm{min}]$

FIG. 2. Changes in the AC spectrogram, DC potentials, cortical temperatures, and cerebral hemodynamics for GS1. In the trace of cerebral hemodynamics, the changes in $\mathrm{HbO}_{2}, \mathrm{HHb}$, and $\mathrm{HbT}$ are indicated as red, blue, and black lines, respectively. A short-term power increase in the AC spectrogram, a series of highamplitude slow waves of $\mathrm{DC}$, a decrease in cortical temperature followed by an increase in the cortical temperature, an increase in $\mathrm{HbO}_{2}$ and $\mathrm{HbT}$ with a decrease in $\mathrm{HHb}$, followed by a decrease in $\mathrm{HbO}_{2}$ and $\mathrm{HbT}$ with an increase in $\mathrm{HHb}$, and a return to the baseline were observed.

the pathophysiological signals obtained during FS1 and FS2 and processed using the Butterworth figure are shown in Fig. 5. These pathophysiological signals are displayed for 10 minutes, which ranges from 5 minutes before onset to 5 minutes after onset. The time corresponding to the seizure onset was set to $t=0$ and highlighted with the green line marked "Onset" in Figs. 2-5.

For GSs, a change in the AC spectrogram obtained from channel 1 (ch1) is shown at the top of Figs. 2-4. A short-term power increase of $20 \mathrm{~Hz}$ or less was observed at the onset of GS1 and GS3. However, no obvious change in power was observed for GS2.

The change in $D C$ is shown in the second row of Figs. 2-4. DCs measured from ch1, ch2, ch3, ch4, ch5, and ch6 are indicated as red, pink, yellow, light green, light blue, and blue lines, respectively. A series of high-amplitude slow waves was observed in all the seizures. The change in DC lasted for more than 2 minutes in all seizures and began approximately 30 seconds before the onset of GS1 and GS3. 

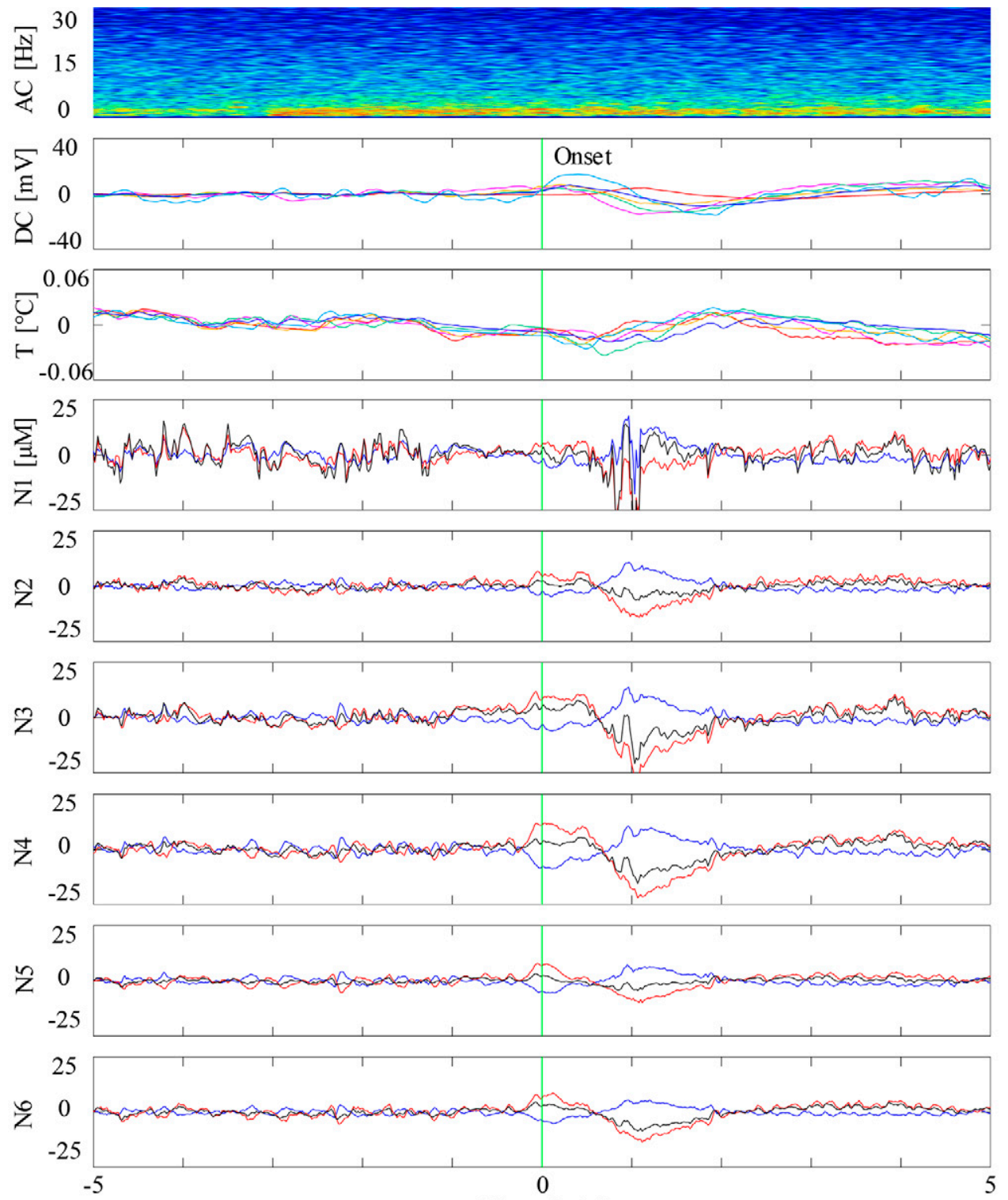

Time $[\mathrm{min}]$

FIG. 3. Changes in the AC spectrogram, DC potentials, cortical temperatures, and cerebral hemodynamics for GS2. In the trace of cerebral hemodynamics, the changes in $\mathrm{HbO}_{2}, \mathrm{HHb}$, and $\mathrm{HbT}$ are indicated as red, blue, and black lines, respectively. Although no obvious increase in power could be observed in the AC spectrogram, a series of high-amplitude slow waves of $\mathrm{DC}$, a decrease in cortical temperature followed by an increase in cortical temperature, an increase in $\mathrm{HbO}_{2}$ and $\mathrm{HbT}$ with a decrease in $\mathrm{HHb}$, followed by a decrease in $\mathrm{HbO}_{2}$ and $\mathrm{HbT}$ with an increase in $\mathrm{HHb}$, and a return to the baseline were observed.

Changes in the cortical temperature are displayed in the third row of Figs. 2-4. Cortical temperatures measured from ch1, ch2, ch3, ch4, ch5, and ch6 are indicated as red, pink, yellow, light green, light blue, and blue lines, respectively, similar to the DCs. An increase in cortical temperature was observed in all the GSs. However, an initial decrease followed by an increase in the cortical temperature was observed for GS1 and GS2. The waveforms of the cortical temperature were consistent across all the channels.

Changes in the cerebral hemodynamics were displayed in order from ch1 to ch6, from the fourth row to the ninth row in Figs. 2-4. The changes in $\mathrm{HbO}_{2}, \mathrm{HHb}$, and $\mathrm{HbT}$ are indicated as red, blue, and black lines, respectively. The waveforms of $\mathrm{HbO}_{2}, \mathrm{HHb}$, and $\mathrm{HbT}$ are disturbed in the case of GS3. However, an increase in $\mathrm{HbO}_{2}$ and $\mathrm{HbT}$ with a decrease in $\mathrm{HHb}$, followed by a decrease in $\mathrm{HbO}_{2}$ and $\mathrm{HbT}$ with an increase in $\mathrm{HHb}$, and a return to the baseline were observed in all the GSs. These changes started approximately 30 seconds before the onset in GS1 and GS2.

For FSs, the changes in the pathophysiological signals were not observed using the multimodal probe (Fig. 5).

Changes in DC, cortical temperature, and cerebral hemodynamics for GS1, GS2, GS3, FS1, and FS2, without the Butterworth filter, are presented in detail in Supplemental Figs. 1-5, respectively, similar to 

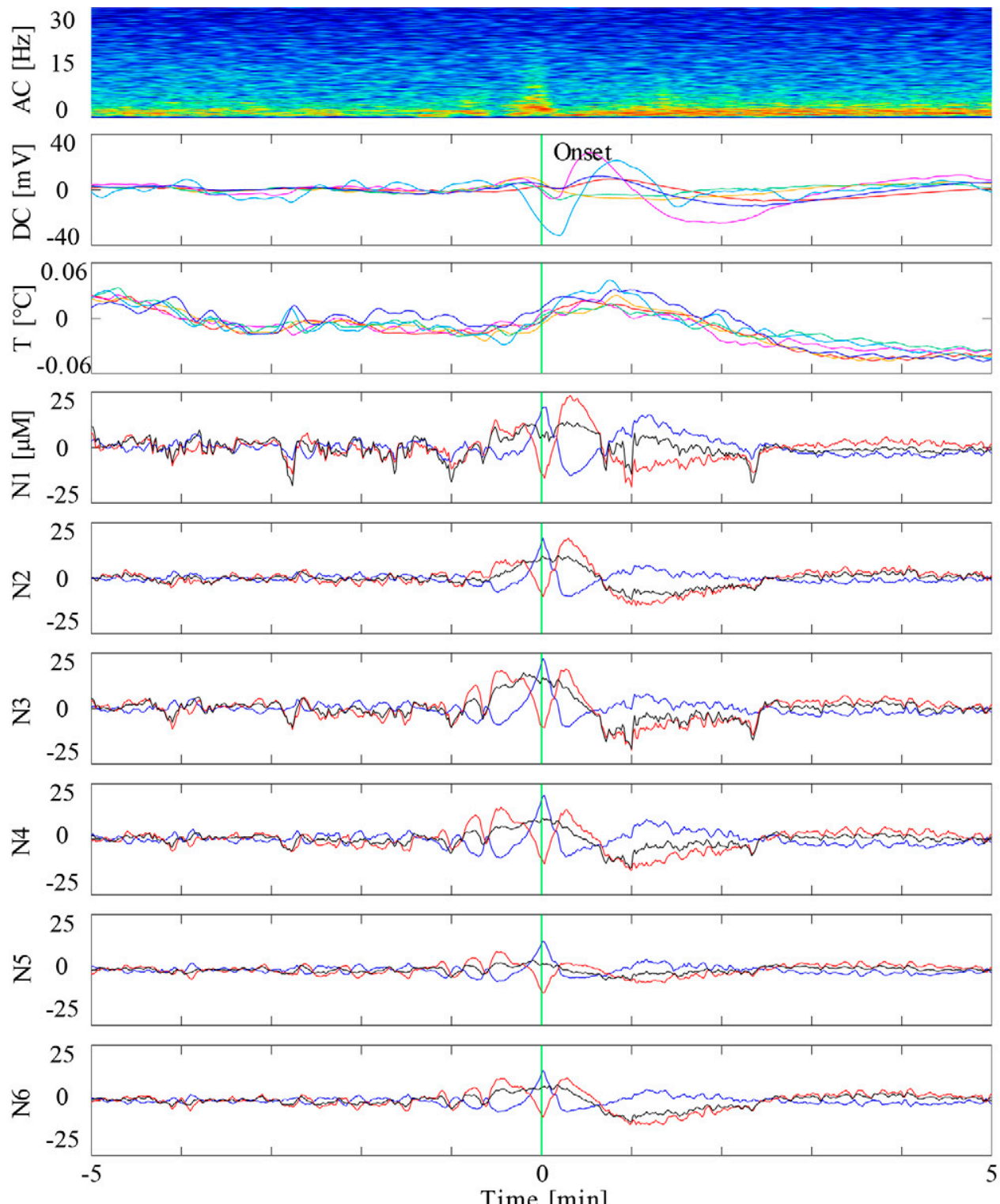

Time [min]

FIG. 4. Changes in the AC spectrogram, DC potentials, cortical temperatures, and cerebral hemodynamics for GS3. In the trace of cerebral hemodynamics, the changes in $\mathrm{HbO}_{2}, \mathrm{HHb}$, and $\mathrm{HbT}$ are indicated as red, blue, and black lines, respectively. A short-term power increase in the AC spectrogram, a series of highamplitude slow waves of DC, and an increase in cortical temperature were observed. Regarding the cerebral hemodynamics, although the waveforms were disturbed, waveforms similar to an increase in $\mathrm{HbO}_{2}$ and $\mathrm{HbT}$ with a decrease in $\mathrm{HHb}$, followed by a decrease in $\mathrm{HbO}_{2}$ and $\mathrm{HbT}$ with an increase in $\mathrm{HHb}$, and a return to the baseline were observed.

Figs. 2-4. Although there was a baseline fluctuation, seizure-related changes in DC, cortical temperature, and cerebral hemodynamics were observed without the Butterworth filter in GSs (Supplemental Figs. 1-3). No change in these pathophysiological signals was observed in FSs (Supplemental Figs. 4 and 5), similar to filtered signals obtained during FSs. In addition, it could be considered that the filtered signals contain seizure-related changes and the Butterworth filter successfully emphasized seizure-related changes by comparing figures processed using the Butterworth filter (Figs. 2-5) with figures processed without the Butterworth filter (Supplemental Figs. 1-5).

\section{Discussion}

\section{Observations}

A short-term power increase was observed in the AC spectrogram for GS1 and GS3. This power increase indicates that an epileptic discharge was obtained from the proposed probe. However, no evident change was noted for GS2. This could be because the epileptic discharge did not propagate to the measurement area in GS2, as the developed probe was not placed in the vicinity of the epileptic focus.

As a change in DC, a slow potential composed of upward or downward fluctuation of $0.0025-0.01 \mathrm{~Hz}$ was observed. This result 

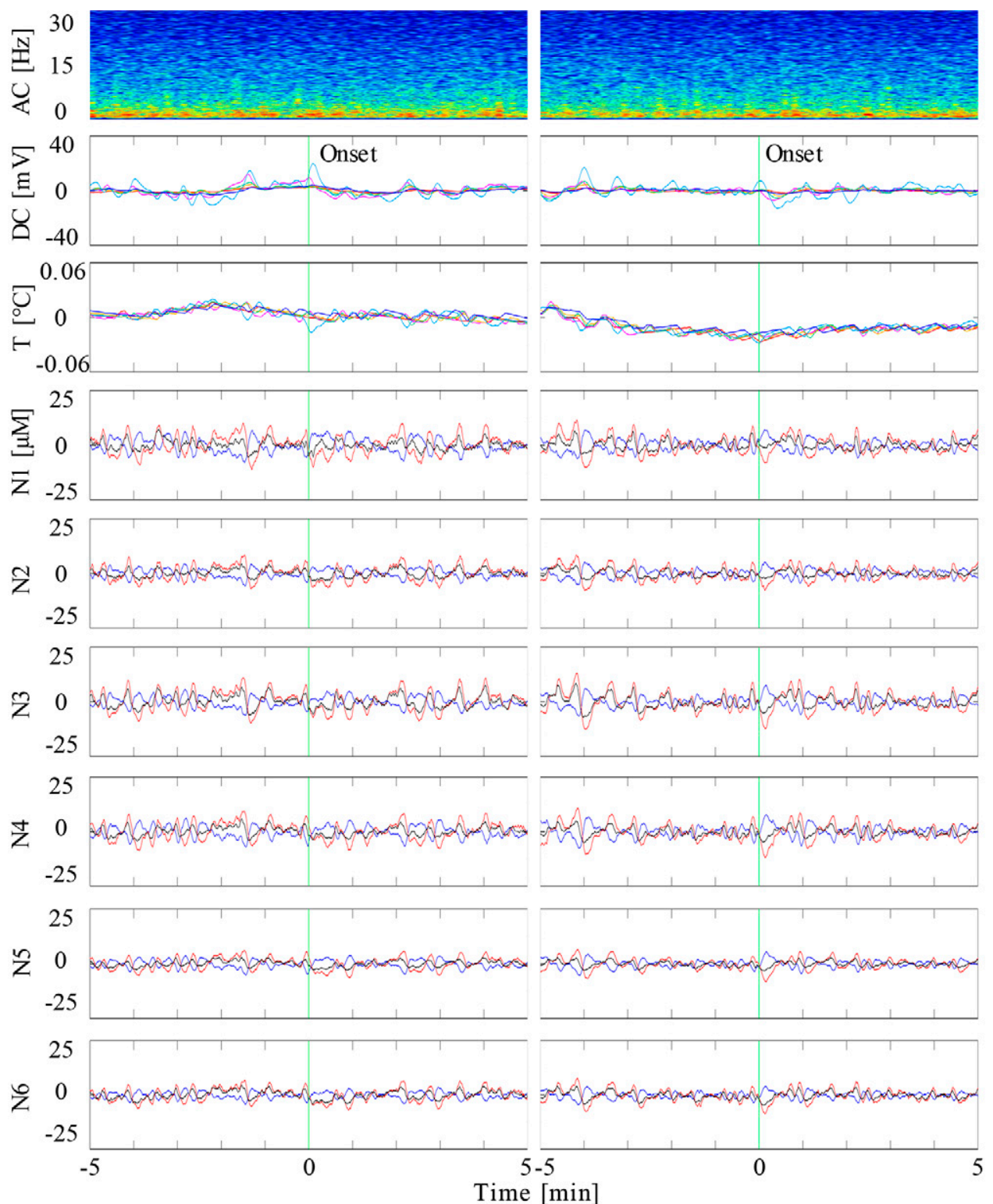

FIG. 5. Changes in the AC spectrogram, DC potentials, cortical temperatures, and cerebral hemodynamics during FSs. Left and right figures show changes in pathophysiological signals for FS1 and FS2, respectively. No changes in the pathophysiological signals were recorded with the multimodal probe.

was similar to the "DC shift," which is defined as slow potential and composed of an upward or downward phase of $0.016-0.03 \mathrm{~Hz}$ that lasts at least 3 seconds. ${ }^{4}$

The brain temperature is maintained by balancing the heat production and removal within the brain. Heat is produced by oxygen consumption and removed by the cerebral blood flow (CBF)..$^{15}$ In this study, an increase in the cortical temperature was noted in all the seizures. This temperature elevation is similar to the result of a study which found that the activation of neural metabolism related to epileptic seizures increases the cortical temperature in epileptic rats induced by bicuculline methiodide. ${ }^{5}$ In addition, it was found that the elevation of brain temperature accelerates the epileptic discharge in an epileptic mouse model induced by kindling stimuli. ${ }^{6}$
However, in the case of the GSs, GS1 and GS2, the cortical temperature slightly decreased before it increased. It could be considered that the decline in the cortical temperature may have been caused by the increased CBF. In humans, the brain temperature is higher than the core body temperature. ${ }^{16}$ Moreover, the increased CBF caused by epileptic seizures decreases the brain temperature in case of no heat exchange with the environment. ${ }^{17}$

As for the change in the cerebral hemodynamics, the change in $\mathrm{HbT}$ reflects the local cerebral blood volume, ${ }^{7}$ and the changes in $\mathrm{HbO}_{2}$ and $\mathrm{HHb}$ are determined on the basis of balance between the oxygen consumption and $\mathrm{CBF}$, which carries hemoglobin. ${ }^{18}$ Change in cerebral hemodynamics consisted of two phases: (1) an increase in $\mathrm{HbO}_{2}$ and $\mathrm{HbT}$ with a decrease in $\mathrm{HHb}$ and (2) a decrease in $\mathrm{HbO}_{2}$ 
and $\mathrm{HbT}$ with an increase in $\mathrm{HHb}$. These changes were similar to those observed in the patients with epilepsy. ${ }^{13}$ An increase in $\mathrm{HbO}_{2}$ and $\mathrm{HbT}$ accompanied by a decrease in $\mathrm{HHb}$ indicates that $\mathrm{HbO}_{2}$ was carried to the brain by an increase in CBF in response to the increased oxygen consumption due to epileptic seizures. ${ }^{7,8}$ Conversely, a decrease in $\mathrm{HbO}_{2}$ with an increase in $\mathrm{HHb}$ suggests that the oxygen consumption could be increased and could not be sufficiently compensated by the increased CBF. ${ }^{13,19}$

Although no obvious change in the $A C$ spectrogram was observed in GS2, seizure-related changes in DC, cortical temperature, and cerebral hemodynamics were observed in all GSs. However, changes in these pathophysiological signals have not been observed in FSs. These results indicate that epileptic brain activity propagated from the epileptic focus in the right frontal lobe to the measurement site near the motor cortex, far from the epileptic focus in GSs, but not in FSs. These were consistent with the diagnostic outcomes of the GSs or FSs.

As for clinical follow-up, one of the cortical tubers responsible for the seizure was resected along with the surrounding epileptogenic zone, which was defined by video EEG monitoring. Postoperatively, the patient was seizure free for the first half year; however, due to another cortical tuber, a focal onset seizure was restarted. No adverse event related to the present study was detected.

\section{Lessons}

The multimodal probe obtained changes in EcoG, cerebral hemodynamics, and cortical temperature related to GSs, and these changes were consistent with the previous studies. However, these seizure-related changes were not observed in FSs. This result was concordant with the diagnostic outcomes of the GSs or FSs. In addition, for a single GS, the proposed probe obtained changes in DC, cortical temperature, and cerebral hemodynamics, even though no evident change in AC was observed. This result indicates that the proposed probe minimizes the risk of missing a seizure by measuring multiple signals simultaneously. The limitations of this study are the probe location and the number and age of participants. The epileptic focus was located in the right frontal lobe, whereas the developed probe was installed near the right motor cortex so as not to interfere with the diagnosis. The measurements from the vicinity of the epileptic focus are required to observe the change in pathophysiological signals at the epileptic focus. In addition, the participant in this study was only one pediatric patient. Measurements from participants of other ages may yield different observations due to the difference in the degree of the development of the brain. Further studies with larger numbers of participants of different ages are required for a more in-depth analysis of the changes in the pathophysiological signals associated with epileptic seizures and to demonstrate the clinical significance of the proposed probe.

\section{Acknowledgments}

Funding was awarded to Michiyasu Suzuki by the Japan Society for the Promotion of Science (JSPS) (grant nos. $15 \mathrm{H} 05719$ and 21H03841) and Ministry of Education, Culture, Sports, Science and Technology (MEXT).

\section{References}

1. Alarcon G, Garcia Seoane JJ, Binnie CD, et al. Origin and propagation of interictal discharges in the acute electrocorticogram. Implications for pathophysiology and surgical treatment of temporal lobe epilepsy. Brain. 1997;120(Pt 12):2259-2282.

2. Hufnagel A, Dümpelmann M, Zentner J, Schijns O, Elger CE. Clinical relevance of quantified intracranial interictal spike activity in presurgical evaluation of epilepsy. Epilepsia. 2000;41(4):467-478.

3. Zumsteg D, Wieser HG. Presurgical evaluation: current role of invasive EEG. Epilepsia. 2000;41(Suppl 3):S55-S60.

4. Ikeda A, Taki W, Kunieda T, et al. Focal ictal direct current shifts in human epilepsy as studied by subdural and scalp recording. Brain. 1999;122(Pt 5):827-838.

5. He $\mathrm{Y}$, Inoue T, Nomura S, et al. Limitations of local brain cooling on generalized motor seizures from unknown foci in awake rats. Neurol Med Chir (Tokyo). 2019;59(4):147-153.

6. Shibasaki K, Yamada K, Miwa H, et al. Temperature elevation in epileptogenic foci exacerbates epileptic discharge through TRPV4 activation. Lab Invest. 2020;100(2):274-284.

7. Roche-Labarbe N, Zaaimi B, Mahmoudzadeh M, et al. NIRS-measured oxy- and deoxyhemoglobin changes associated with EEG spike-and-wave discharges in a genetic model of absence epilepsy: the GAERS. Epilepsia. 2010;51(8):1374-1384.

8. Roche-Labarbe N, Zaaimi B, Berquin P, Nehlig A, Grebe R, Wallois F. NIRS-measured oxy- and deoxyhemoglobin changes associated with EEG spike-and-wave discharges in children. Epilepsia. 2008:49(11):1871-1880.

9. Nguyen DK, Tremblay J, Pouliot $P$, et al. Noninvasive continuous functional near-infrared spectroscopy combined with electroencephalography recording of frontal lobe seizures. Epilepsia. 2013;54(2): 331-340.

10. Yang XF, Chang JH, Rothman SM. Intracerebral temperature alterations associated with focal seizures. Epilepsy Res. 2002;52(2): 97-105.

11. Hoshi $\mathrm{Y}$, Kobayashi N, Tamura M. Interpretation of near-infrared spectroscopy signals: a study with a newly developed perfused rat brain model. J Appl Physiol (1985). 2001;90(5):1657-1662.

12. Gallagher A, Lassonde M, Bastien D, et al. Non-invasive pre-surgical investigation of a 10 year-old epileptic boy using simultaneous EEG-NIRS. Seizure. 2008;17(6):576-582.

13. Nguyen DK, Tremblay J, Pouliot $P$, et al. Non-invasive continuous EEG-fNIRS recording of temporal lobe seizures. Epilepsy Res. 2012;99(1-2):112-126.

14. Yamakawa T, Inoue T, Niwayama M, et al. Implantable multi-modality probe for subdural simultaneous measurement of electrophysiology, hemodynamics, and temperature distribution. IEEE Trans Biomed Eng. 2019;66(11):3204-3211.

15. Yablonskiy DA, Ackerman JJ, Raichle ME. Coupling between changes in human brain temperature and oxidative metabolism during prolonged visual stimulation. Proc Natl Acad Sci U S A. 2000;97(13):7603-7608.

16. Wang $H$, Wang $B$, Normoyle $K P$, et al. Brain temperature and its fundamental properties: a review for clinical neuroscientists. Front Neurosci. 2014;8:307.

17. Sukstanskii AL, Yablonskiy DA. Theoretical model of temperature regulation in the brain during changes in functional activity. Proc Natl Acad Sci U S A. 2006;103(32):12144-12149.

18. Schridde U, Khubchandani M, Motelow JE, Sanganahalli BG, Hyder $F$, Blumenfeld $H$. Negative BOLD with large increases in neuronal activity. Cereb Cortex. 2008;18(8):1814-1827.

19. Zhao M, Suh M, Ma H, Perry C, Geneslaw A, Schwartz TH. Focal increases in perfusion and decreases in hemoglobin oxygenation precede seizure onset in spontaneous human epilepsy. Epilepsia. 2007;48(11):2059-2067.

\section{Disclosures}

Dr. Inoue reported a patent for Japan Patent No. 6296606 (licensed) and a patent for Japan Patent Application No. 2020-112158 (pending). 
Dr. Nomura reported a patent issued (201503004750105776). Dr Yamakawa reported a patent issued (JP6460457) for Kumamoto University. The other authors report no conflict of interest concerning the materials or methods used in this study or the findings specified in this paper.

\section{Author Contributions}

Conception and design: Yamakawa, Inoue, Suzuki. Acquisition of data: Yamakawa, Inoue, Imoto, Nomura, Suzuki. Analysis and interpretation of data: Wakuya, Suzuki. Drafting the article: Wakuya, Suzuki. Critically revising the article: Yamakawa, Inoue. Reviewed submitted version of manuscript: Yamakawa, Inoue. Approved the final version of the manuscript on behalf of all authors: Yamakawa. Administrative/ technical/material support: Imoto, Maruta. Study supervision: Yamakawa.

\section{Supplemental Information}

Online-Only Content

Supplemental material is available with the online version of the article. Supplemental Figures 1-5. http://thejns.org/doi/suppl/10.3171/ CASE21694.

\section{Correspondence}

Toshitaka Yamakawa: Faculty of Advanced Science and Technology, Kumamoto University, Kumamoto, Japan. yamakawa@cs.kumamoto-u. ac.jp. 